\title{
PROFIT MAXIMIZATION MULTI-ITEM INVENTORY MODELS CONSIDERING TRADE CREDIT AND SALES LEARNING CURVE
}

\author{
Yu-Chung Tsao \\ Department of Business Management, Tatung University \\ Taipei 104, Taiwan. \\ yctsao@ttu.edu.tw
}

\begin{abstract}
In this paper we consider a multi-item distribution channel subject to supplier's credit period and retailer's promotional effort with sales learning curve. We determine the retailer's promotional effort for each item and the joint replenishment cycle in both decentralized and centralized decision models. We show that the profits for both parties increase under the centralized decision model when the credit period is kept within an appropriate range. The impacts of credit period and sales learning curve on the behavior of the channel are also discussed. We conclude with computation analyses that lead to a variety of management insights.
\end{abstract}

Key Words- Inventory, Sales learning curve, Promotion, Credit period

\section{INTRODUCTION}

Retailer promotional activity has become more and more common in today's business world. For example, McDonald's and Burger King use coupons to stimulate demand; Wall-Mart and Costco provide price discount for specific electric equipment to stimulate its demand. Krishnam et al. [1] stated that the promotional strategies include price cuts, displays, free goods, and advertising. Too many promotions may incur residual costs and too few may result in lower profit. Therefore, the promotion policy is very important for the retailer. This paper incorporates the retailer's promotion effort decision into an inventory model.

In the real world replenishment and inventory control are truly large scale problems, often involving dozens or even hundreds of items. In a multi-item distribution channel, considerable savings can be realized during the replenishment by coordinating the ordering of several different items. Joint multi-item replenishment strategies have been widely applied in the real world, for example, the supplying of parts for automotive assembly (Hahm and Yano [2]) or of refrigerated goods to supermarkets (Hammer [3]). Several researchers (e.g. Rempala [4], Chen and Chen [5]) have proposed models and algorithms for solving multi-item replenishment problems for different situations.

In practice, the supplier often provides forward financing to the retailer. Goyal [6], Chang et al. [7], and Teng [8] determined the optimal ordering policy from the retailer's perspective for different situations, given a credit period. From the perspective of suppliers, Kim et al. [9], Abad and Jaggi [10], and Sheen and Tsao [11] determined the optimal credit policy needed to maximize supplier profit with price-dependent demand functions. It is essential to consider the practice of trade credit when formulating a decision-making model. However, all the above researchers considered only the singleitem inventory problem, ignoring the effect of promotion and multi-item replenishment. 
In addition, sales learning curve has been discussed by Leslie and Holloway [12]. They examined the concept of sales learning curve: The more times a process is repeated, the more efficient it becomes and the lower its cost. This means that the promotion cost will decrease over time in this model. The main contributions of this paper to the literature include: First, this is the first study to consider the supplier's credit period and retailer multi-item replenishment and promotion policies. Second, this is the first study to incorporate the idea of sales learning curve into the promotion cost. Third, this paper utilizes the credit period as a mechanism to increase supplier's and retailer's profits and shows the distribution of the increasable profit.

The following notations are used in this study. $r_{i}$ : retail price for item $i, w_{i}$ : wholesale price for item $i, c_{i}$ : the supplier's purchase cost for item $i, A_{j}$ : major setup cost per order, where $j=R$ for retailer and $j=S$ for supplier, $a_{j, i}$ : minor setup cost for adding item $i$ into the order, where $j=R$ for retailer and $j=S$ for supplier, $H_{j, i}$ : holding cost for item $i$, where $j=R$ for retailer and $j=S$ for supplier, $T$ : replenishment cycle, $q_{i}$ : replenishment quantity for item $i, \rho_{i}$ : retailer promotional effort for item $i, \rho_{i} \geq 1, \xi_{i}$ : basic demand for item $i, t$ : credit period in the decentralized decision model, $t^{\prime}$ : credit period in the centralized decision model, $h$ : the interest rate of short-term capital

$C_{i}\left(\rho_{i}, \xi_{i}\right)$ : annual promotional effort cost for item $i, \Pi_{j}$ : annual profit, where $j=R$ for the retailer, $j=S$ for the supplier and $j=C$ for the channel.

The mathematical model is developed under the following assumptions: Firstly, the promotional effort $\rho_{i}$ for item $i$ does not affect the basic demand $\xi_{i}$, but affects the effort-induced demand $\rho_{i} \xi_{i}$. Secondly, the promotional effort cost for item $i$ at time $t$ $\left(v_{i}(t)=K_{i}\left(\rho_{i}-1\right)^{2} \xi_{i} \alpha_{i} e^{-\theta_{i} t}\right.$, where $K_{i}>0, \theta_{i}>0$ and $\alpha_{i}$ are constants) decreases in time with rate $e^{-\theta_{i} t}$, i.e. the effect of sales learning curve. This form is modified from Krishnam et al. (2004) by incorporating the learning effect. Higher values of $\theta_{i}$ imply higher effect of sales learning with a lower promotion cost. A higher value of $K_{i}$ or $\alpha_{i}$ means a greater difficulty in attracting customers. The promotional effort cost from time $t_{1}$ to time $t_{2}$ for item $i$ is $C_{i}^{\left(t_{1}, t_{2}\right)}=K_{i}\left(\rho_{i}-1\right)^{2} \xi_{i}^{\alpha_{i}} \int_{t_{1}}^{t_{2}} e^{-\theta_{i} t} d t$, which is illustrated in Figure 1. Given $C_{i}^{(0,1)}$, assuming the initial time $t_{1}=0$ and the end time $t_{2}=1$ in this model, we obtain $C_{i}=C_{i}^{(0,1)}=K_{i}\left(\rho_{i}-1\right)^{2} \xi_{i}^{\alpha_{i}} \frac{1-e^{-\theta_{i}}}{\theta_{i}}$. Finally, the supplier provides a credit period $t$ to the retailer, and a capital opportunity cost due to the period incurs. The retailer gains a capital opportunity benefit due to the credit period. 


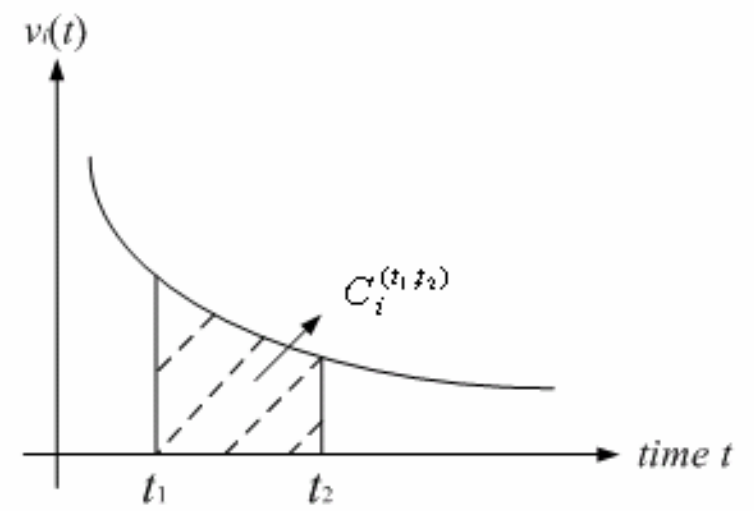

Figure 1. Graphic representations of $C_{i}^{\left(t_{1}, t_{2}\right)}$ from time $t_{1}$ to time $t_{2}$ for item $i$.

\section{DECENTRALIZED DECISION MODEL}

In the decentralized decision model, the retailer determines the promotion and replenishment decisions to maximize her/his profit. The retailer's profit is:

$$
\Pi_{R}\left(\rho_{i}, T\right)=-\frac{A_{R}}{T}+\sum_{i=1}^{n}\left[\begin{array}{l}
\left(r_{i}-w_{i}\right) \rho_{i} \xi_{i}-\frac{a_{R, i}}{T}-\frac{\rho_{i} \xi_{i} T}{2}\left(H_{R, i}+w_{i} h\right) \\
-K_{i}\left(\rho_{i}-1\right)^{2} \xi_{i} \alpha_{i} \frac{1-e^{-\theta_{i}}}{\theta_{i}}+w_{i} \rho_{i} \xi_{i} t h
\end{array}\right] .
$$

The supplier's profit is given by

$$
\Pi_{S}\left(\rho_{i}, T\right)=-\frac{A_{S}}{T}+\sum_{i=1}^{n}\left[\left(w_{i}-c_{i}\right) \rho_{i} \xi_{i}-\frac{a_{S, i}}{T}-\frac{\rho_{i} \xi_{i} T}{2}\left(H_{S, i}+c_{i} h\right)-w_{i} \rho_{i} \xi_{i} t h\right] .
$$

The retailer determines her/his optimal promotional effort $\rho_{i}$ for item $i$ and the replenishment cycle $T$. We use the following lemma to discuss the optimal solution.

Lemma 1. The solution of $\frac{\partial \Pi_{R}\left(\rho_{i}, T\right)}{\partial \rho_{i}}=0, i=1,2, \ldots, n$, and $\frac{\partial \Pi_{R}\left(\rho_{i}, T\right)}{\partial T}=0$ is maximal for $\Pi_{R}\left(\rho_{i}, T\right)$.

Proof: $\frac{\partial^{2} \Pi_{R}\left(\rho_{i}, T\right)}{\partial T^{2}}=-\frac{2\left(A_{R}+\sum_{i=1}^{n} a_{R, i}\right)}{T^{3}}<0, \frac{\partial^{2} \Pi_{R}\left(\rho_{i}, T\right)}{\partial \rho_{i}{ }^{2}}=-2 K_{i} \xi_{i}{ }^{\alpha_{i}} \frac{1-e^{-\theta_{i}}}{\theta_{i}}<0$ for item $i, \frac{d^{2} \Pi_{R}\left(\rho_{i}, T\right)}{d T d \rho_{i}}=-\frac{\xi_{i} \cdot\left(H_{R, i}+w_{i} h\right)}{2}+t c \cdot h \cdot w_{i}$ for item $i, i=1,2, \ldots, n$. Then the Hessian matrix is 


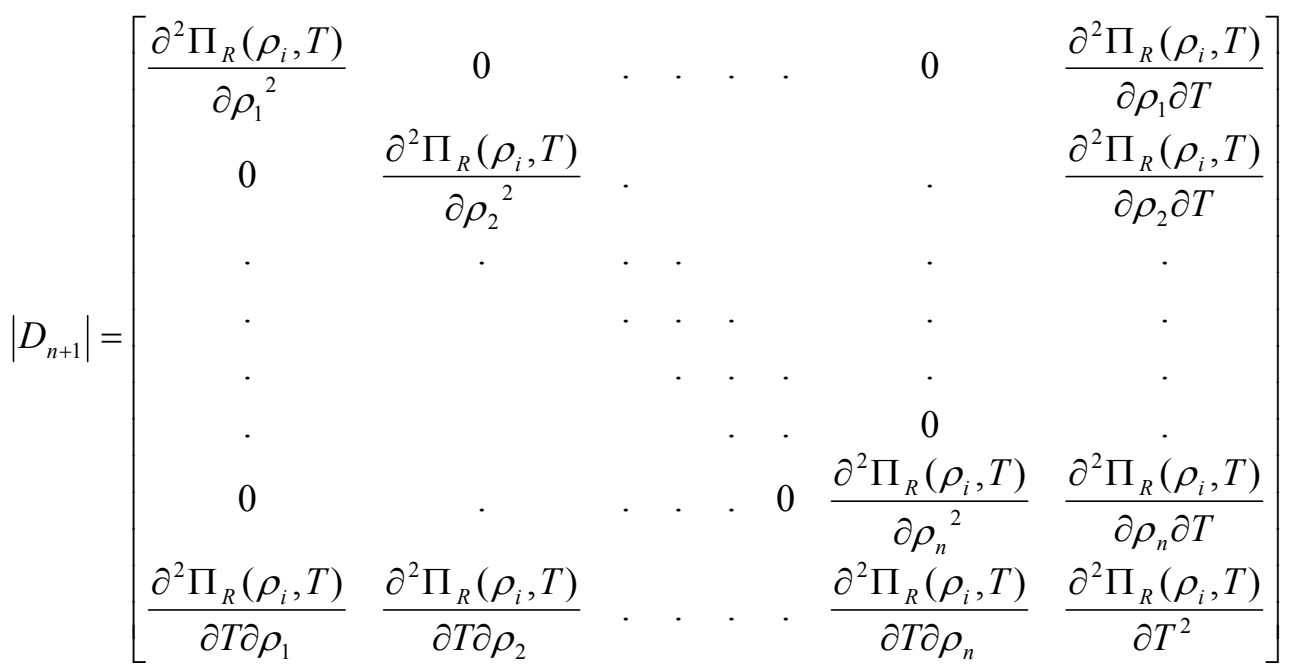

Since $\frac{\partial^{2} \Pi_{R}\left(\rho_{i}, T\right)}{\partial T^{2}}<0$ and $\frac{\partial^{2} \Pi_{R}\left(\rho_{i}, T\right)}{\partial \rho_{i}{ }^{2}}<0$ for all $i$, we know $(-1)^{i} \cdot\left|D_{i}\right|>0$ is satisfied for all $i, i=1,2, \ldots, n$. From Winston [13], this means that there must exists a solution of $\frac{\partial \Pi_{R}\left(\rho_{i}, T\right)}{\partial \rho_{i}}=0, i=1,2, \ldots, n$, and $\frac{\partial \Pi_{R}\left(\rho_{i}, T\right)}{\partial T}=0$ to $\operatorname{maximize} \Pi_{R}\left(\rho_{i}, T\right)$.

Let $\rho_{i}^{t}$ and $T^{t}$ be the optimal promotional effort and replenishment cycle to maximize the retailer's profit. From $\frac{d \Pi_{R}\left(\rho_{i}, T\right)}{d T}=0$, we obtain

$$
T^{t}\left(\rho_{i}\right)=\sqrt{\frac{2\left(A_{R}+\sum_{i=1}^{n} a_{R, i}\right)}{\sum_{i=1}^{n} \rho_{i} \xi_{i}\left(H_{R, i}+w_{i} h\right)}} .
$$

Equation (3) is the optimal replenishment cycle in the decentralized decision model when all promotional efforts are given. The replenishment quantity for item $i$ is $\rho_{i}^{t} \xi_{i} T^{t}$.

\section{CENTRALIZED DECISION MODEL}

In the centralized decision model, the promotional effort and the replenishment cycle are determined to maximize the channel's profit. The channel's profit is defined as the sum of the retailer's profit and the supplier's profit as

$$
\begin{aligned}
\Pi_{C}\left(\rho_{i}, T\right)=-\frac{A_{R}+A_{S}}{T}+\sum_{i=1}^{n}\left[\begin{array}{l}
\left(r_{i}-c_{i}\right) \rho_{i} \xi_{i}-\frac{a_{R, i}+a_{S, i}}{T}-\frac{\rho_{i} \xi_{i} T}{2} \cdot\left(I_{R, i}+I_{S, i}\right) \\
-K_{i}\left(\rho_{i}-1\right)^{2} \xi_{i} \alpha_{i} \frac{1-e^{-\theta_{i}}}{\theta_{i}}
\end{array}\right], \\
\text { where } I_{R, i}=H_{R, i}+w_{i} h \text { and } I_{S, i}=H_{S, i}+c_{i} h .
\end{aligned}
$$

$I_{R, i}\left(I_{S, i}\right)$ is the retailer (supplier) unit inventory cost for item $i$ (Sheen and Tsao (2007)). The retailer determines the optimal promotional effort for each item and the 
joint replenishment cycle. We use Lemma 2 to determine the maximum value of the promotional effort and the replenishment cycle.

Lemma 2. The solution of $\frac{\partial \Pi_{C}\left(\rho_{i}, T\right)}{\partial \rho_{i}}=0, i=1,2, \ldots, n$, and $\frac{\partial \Pi_{C}\left(\rho_{i}, T\right)}{\partial T}=0$ is maximal for $\Pi_{C}\left(\rho_{i}, T\right)$.

Proof: Since $\frac{\partial^{2} \Pi_{C}\left(\rho_{i}, T\right)}{\partial T^{2}}=-\frac{2\left[A_{R}+A_{S}+\sum_{i=1}^{n}\left(a_{R, i}+a_{S, i}\right)\right]}{T^{3}}<0 \quad, \quad \frac{\partial^{2} \Pi_{C}\left(\rho_{i}, T\right)}{\partial \rho_{i}^{2}}=$ $-2 K_{i} \xi_{i} \alpha_{i} \frac{1-e^{-\theta}}{\theta}<0$ for item $i, \frac{d^{2} \Pi_{C}\left(\rho_{i}, T\right)}{d T d \rho_{i}}=-\frac{\xi_{i}\left(I_{R, i}+I_{S, i}\right)}{2}<0$ for item $i$, $i=1,2, \ldots, n$. The proof is similar to that in Lemma 1 .

Let $\rho_{i}^{I}$ and $T^{I}$ be the optimal promotional effort and replenishment cycle to maximize the channel's profit. From $\frac{d \Pi_{C}\left(\rho_{i}, T\right)}{d T}=0$, we obtain

$$
T^{I}\left(\rho_{i}\right)=\sqrt{\frac{2\left[A_{R}+A_{S}+\sum_{i=1}^{n}\left(a_{R, i}+a_{S, i}\right)\right]}{\sum_{i=1}^{n} \rho_{i} \xi_{i}\left(I_{R, i}+I_{S, i}\right)}}
$$

Equation (5) is the optimal replenishment cycle in the centralized decision model when promotional effort for each item is given. And the replenishment quantity for item $i$ is $\rho_{i}^{I} \xi_{i} T^{I}$.

After showing retailer's decisions in both decentralized and centralized decision models, Proposition 1 illustrates the channel profit while the retailer adopts $\rho_{i}^{I}$ and $T^{I}$ higher than that while the retailer adopts $\rho_{i}^{t}$ and $T^{t}$.

\section{Proposition 1.}

(a) Given $\rho_{i}$ for each item, $\Pi_{R}\left(T^{I}, \rho_{i}\right)+\Pi_{S}\left(T^{I}, \rho_{i}\right) \geq \Pi_{R}\left(T^{t}, \rho_{i}\right)+\Pi_{S}\left(T^{t}, \rho_{i}\right)$.

(b) $\Pi_{R}\left(\rho_{i}^{I}, T^{I}\right)+\Pi_{S}\left(\rho_{i}^{I}, T^{I}\right) \geq \Pi_{R}\left(\rho_{i}^{t}, T^{t}\right)+\Pi_{S}\left(\rho_{i}^{t}, T^{t}\right)$.

Proof: (a) Substituting equation (3) into equations (1) and (2), we obtain $\Pi_{R}\left(T^{t}, \rho_{i}\right)+\Pi_{S}\left(T^{t}, \rho_{i}\right)$

$$
\begin{aligned}
& =\sum_{i=1}^{n}\left[\left(r_{i}-c_{i}\right) \rho_{i} \xi_{i}-K_{i}\left(\rho_{i}-1\right)^{2} \xi_{i} \alpha_{i} \frac{1-e^{-\theta_{i}}}{\theta_{i}}\right]-\left(\frac{A_{S}+\sum_{i=1}^{n} a_{S, i}}{A_{R}+\sum_{i=1}^{n} a_{R, i}}+\frac{\sum_{i=1}^{n} I_{S, i}}{\sum_{i=1}^{n} I_{R, i}}\right) \times \\
& \sqrt{\frac{\rho_{i} \xi_{i}\left(A_{R}+\sum_{i=1}^{n} a_{R, i}\right)\left(\sum_{i=1}^{n} I_{R, i}\right)}{2}}-\sqrt{2 \rho_{i} \xi_{i}\left(A_{R}+\sum_{i=1}^{n} a_{R, i}\right)\left(\sum_{i=1}^{n} I_{R, i}\right)} \\
& =\sum_{i=1}^{n}\left[\left(r_{i}-c_{i}\right) \rho_{i} \xi_{i}-K_{i}\left(\rho_{i}-1\right)^{2} \xi_{i}^{\alpha_{i}} \frac{1-e^{-\theta_{i}}}{\theta_{i}}\right]- \\
& 2 \frac{\left(\frac{A_{S}+\sum_{i=1}^{n} a_{S, i}}{A_{R}+\sum_{i=1}^{n} a_{R, i}}+1\right)+\left(\frac{\sum_{i=1}^{n} I_{S, i}}{\sum_{i=1}^{n} I_{R, i}}+1\right)}{2} \cdot \sqrt{\frac{\rho_{i} \xi_{i}\left(A_{R}+\sum_{i=1}^{n} a_{R, i}\right)\left(\sum_{i=1}^{n} I_{R, i}\right)}{2}} .
\end{aligned}
$$


Substituting equation (5) into equations (1) and (2), we obtain

$$
\begin{aligned}
& \Pi_{R}\left(T^{I}, \rho_{i}\right)+\Pi_{S}\left(T^{I}, \rho_{i}\right) \\
& =\sum_{i=1}^{n}\left[\left(r_{i}-c_{i}\right) \rho_{i} \xi_{i}-K_{i}\left(\rho_{i}-1\right)^{2} \xi_{i}^{\alpha_{i}} \frac{1-e^{-\theta_{i}}}{\theta_{i}}\right]-\sqrt{2 \rho_{i} \xi_{i}\left[A_{R}+A_{S}+\sum_{i=1}^{n}\left(a_{R, i}+a_{S, i}\right)\right]} \cdot \sqrt{\sum_{i=1}^{n}\left(I_{R, i}+I_{S, i}\right)} \\
& =\sum_{i=1}^{n}\left[\left(r_{i}-c_{i}\right) \rho_{i} \xi_{i}-K_{i}\left(\rho_{i}-1\right)^{2} \xi_{i} \alpha_{i} \frac{1-e^{-\theta_{i}}}{\theta_{i}}\right]- \\
& 2 \sqrt{\left(\frac{A_{S}+\sum_{i=1}^{n} a_{S, i}}{A_{R}+\sum_{i=1}^{n} a_{R, i}}+1\right) \cdot\left(\frac{\sum_{i=1}^{n} I_{S, i}}{\sum_{i=1}^{n} I_{R, i}}+1\right)} \cdot \sqrt{\frac{\rho_{i} \xi_{i}\left(A_{R}+\sum_{i=1}^{n} a_{R, i}\right)\left(\sum_{i=1}^{n} I_{R, i}\right)}{2}} .
\end{aligned}
$$

From Arithmetic-Geometric Mean Inequality, it is clear that

$$
\frac{\left(\frac{A_{S}+\sum_{i=1}^{n} a_{S, i}}{A_{R}+\sum_{i=1}^{n} a_{R, i}}+1\right)+\left(\frac{\sum_{i=1}^{n} I_{S, i}}{\sum_{i=1}^{n} I_{R, i}}+1\right)}{2} \geq \sqrt{\left(\frac{A_{S}+\sum_{i=1}^{n} a_{S, i}}{A_{R}+\sum_{i=1}^{n} a_{R, i}}+1\right) \cdot\left(\frac{\sum_{i=1}^{n} I_{S, i}}{\sum_{i=1}^{n} I_{R, i}}+1\right)} ;
$$

so $\Pi_{R}\left(T^{I}, \rho_{i}\right)+\Pi_{S}\left(T^{I}, \rho_{i}\right) \geq \Pi_{R}\left(T^{t}, \rho_{i}\right)+\Pi_{S}\left(T^{t}, \rho_{i}\right)$ can be proved.

(b) From (a), we can know $\Pi_{R}\left(T^{I}, \rho_{i}^{t}\right)+\Pi_{S}\left(T^{I}, \rho_{i}^{t}\right) \geq \Pi_{R}\left(T^{t}, \rho_{i}^{t}\right)+\Pi_{S}\left(T^{t}, \rho_{i}^{t}\right)$ for $\rho_{i}=\rho_{i}^{t}$. Since the promotional effort $\rho_{i}^{I}$ is determined to maximize the channel's profit, $\Pi_{R}\left(\rho_{i}^{I}, T^{I}\right)+\Pi_{S}\left(\rho_{i}^{I}, T^{I}\right) \geq \Pi_{R}\left(\rho_{i}^{t}, T^{t}\right)+\Pi_{S}\left(\rho_{i}^{t}, T^{t}\right)$ holds. This completes the proof of (b).

The best condition is that each party will be willing to make decisions from the perspective of the channel when profit will not be sacrificed. The profits of suppliers and the retailers in the decentralized decision model are viewed as the lower bounds for the model in the centralized decision model. Let $\Pi_{S}$ (i.e. $\left.\Pi_{S}\left(\rho_{i}^{t}, T^{t}\right)\right)$ and $\Pi_{R}$ (i.e. $\left.\Pi_{R}\left(\rho_{i}^{t}, T^{t}\right)\right)$ represent the maximal profit of the supplier and the retailer in the decentralized decision model. Propositions 2 and 3 show that profits for both parties increase under the centralized decision model when the credit period is kept within an appropriate range and the distribution of the increasable profit, respectively.

Proposition 2. Profits for both parties increase under centralized decision model when the credit period provided is determined within the appropriate range $\left(t_{\min }, t_{\max }\right)$, where

$$
\begin{aligned}
& t_{\text {min }}=\left\{\Pi_{R}+\frac{A_{R}}{T}-\sum_{i=1}^{n}\left[\left(r_{i}-w_{i}\right) \rho_{i} \xi_{i}-\frac{a_{R, i}}{T}-\frac{\rho_{i} \xi_{i} T}{2} I_{R, i}-K_{i}\left(\rho_{i}-1\right)^{2} \xi_{i} \alpha_{i} \frac{1-e^{-\theta_{i}}}{\theta_{i}}\right]\right\} / \sum_{i=1}^{n} w_{i} \rho_{i} \xi_{i} \\
& \text { and } t_{\text {max }}=\left\{-\frac{A_{S}}{T}-\Pi_{S}+\sum_{i=1}^{n}\left[\left(w_{i}-c_{i}\right) \rho_{i} \xi_{i}-\frac{a_{S, i}}{T}-\frac{\rho_{i} \xi_{i} T}{2} I_{S, i}\right]\right\} / \sum_{i=1}^{n} w_{i} \rho_{i} \xi_{i} h .
\end{aligned}
$$

Proof: From $\Pi_{R}\left(\rho_{i}^{I}, T^{I}\right)-\Pi_{R}>0$ and $\Pi_{S}\left(\rho_{i}^{I}, T^{I}\right)-\Pi_{S}>0$, we can obtain $t_{\min }$ and $t_{\max }$ from $\Pi_{S}\left(\rho_{i}^{I}, T^{I}\right)-\Pi_{S}>0$ respectively. 
Proposition 3. When the credit period is negotiated at $t^{\prime}$, then the channel's increasable profit $\Delta \Pi_{C}^{t^{\prime}}=\left(t_{\max }-t_{\min }\right) \sum_{i=1}^{n} w_{i} \rho_{i} \xi_{i} h$, the supplier's increasable profit $\Delta \Pi_{S}^{t^{\prime}}\left(t^{\prime}\right)=\left(t_{\max }-t^{\prime}\right) \sum_{i=1}^{n} w_{i} \rho_{i} \xi_{i} h$ and the retailer's increasable profit $\Delta \Pi_{R}^{t^{\prime}}\left(t^{\prime}\right)=\left(t^{\prime}-t_{\min }\right) \sum_{i=1}^{n} w_{i} \rho_{i} \xi_{i} h$.

Proof: $\quad t_{\max }-t_{\min }=\left[\left(r_{i}-c_{i}\right) \rho_{i} \xi_{i}-K_{i}\left(\rho_{i}-1\right)^{2} \xi_{i}^{\alpha_{i}} \frac{1-e^{-\theta_{i}}}{\theta_{i}}-\frac{a_{R, i}+a_{S, i}}{T}-\frac{\rho_{i} \xi_{i} T}{2} \cdot\left(I_{R, i}+I_{S, i}\right)\right.$ $\left.-\left(\Pi_{R}+\Pi_{S}\right)\right] / \sum_{i=1}^{n} w_{i} \rho_{i} \xi_{i} h=\left[\left(\Pi_{R}\left(\rho_{i}^{I}, T^{I}\right)+\Pi_{S}\left(\rho_{i}^{I}, T^{I}\right)-\left(\Pi_{R}+\Pi_{S}\right)\right] / \sum_{i=1}^{n} w_{i} \rho_{i} \xi_{i} h\right.$. The proofs of $\Delta \Pi_{S}^{t^{\prime}}\left(t^{\prime}\right)$ and $\Delta \Pi_{R}^{t^{\prime}}\left(t^{\prime}\right)$ are similar to that in $\Delta \Pi_{C}^{t^{\prime}}$.

\section{COMPUTATIONAL ANALYSIS}

We use the following example for item 1 and item 2 to illustrate the proposed model: $r_{1}=9, r_{2}=7, w_{1}=5, w_{2}=4, c_{1}=2, c_{2}=1.5, A_{R}=50, A_{S}=40, a_{R, 1}=a_{R, 2}=1, a_{S, 1}=a_{S, 2}=0.8$, $H_{R, 1}=2, H_{R, 2}=1.5, H_{S, 1}=0.8, H_{S, 2}=0.6, \xi_{1}=1000, \xi_{2}=800, t=0.1, h=0.01, K_{1}=K_{2}=2$, $\alpha_{1}=\alpha_{2}=1.2, \theta_{1}=\theta_{2}=0.05$. The experimental results in the decentralized decision model are: $\rho_{1}^{t}=1.247, \rho_{2}^{t}=1.194, T^{t}=0.1607, \quad q_{1}^{t}=200.418, \quad q_{2}^{t}=153.477, \Pi_{S}=5731.2$, $\Pi_{R}=6519.03$ and $\Pi_{C}=12250.2$. In centralized decision model, $\rho_{1}^{I}=1.435, \rho_{2}^{I}=1.358$, $T^{I}=0.1702, q_{1}^{I}=244.283, q_{2}^{I}=184.935$ and $\Pi_{C}=12689.4$. Figure 2 shows the relationship among the extreme value of credit period and the promotional efforts.

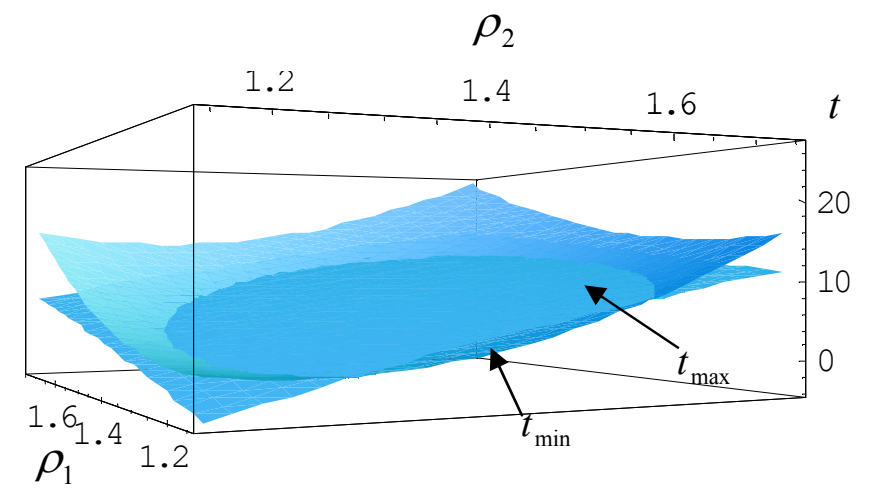

Figure 2. Relationship among credit period and promotional efforts

In Table 1, we compare the individual profit (i.e. $\Pi_{S}$ or $\Pi_{R}$ ) obtained in the decentralized decision model with the corresponding individual profit (i.e. $\Pi_{S}^{t^{\prime}}, \Pi_{R}^{t^{\prime}}$ ) obtained from the decentralized decision model. We observe that the profits of both the suppliers and the retailers increase when $t^{\prime}$ is within $\left(t_{\min }=3.897, t_{\max }=7.709\right)$. The amount of increasable channel profit distributed to the vendor and the buyer will depend on the choice of the credit period $t$. It shows that the profits for both parties increase 
under the centralized decision model when the credit period is kept within an appropriate range

Table 1. Profit distribution under the credit period policy

\begin{tabular}{ccccccc}
\hline$t^{\prime}$ & $\Pi_{S}^{t^{\prime}}$ & $\Pi_{R}^{t^{\prime}}$ & $\Delta \Pi_{S}^{t^{\prime}}$ & $\Delta \Pi_{R}^{t^{\prime}}$ & $\Delta \Pi_{S}^{t^{\prime}} / \Delta \Pi_{C}^{B}$ & $\Delta \Pi_{R}^{t^{\prime}} / \Delta \Pi_{C}^{B}$ \\
\hline \hline 3.896 & 6170.39 & 6519.03 & 439.188 & 0 & 1 & 0 \\
\hline 5.802 & 5950.79 & 6738.62 & 219.594 & 219.594 & 0.5 & 0.5 \\
\hline 7.709 & 5731.2 & 6958.21 & 0 & 439.188 & 0 & 1 \\
\hline
\end{tabular}

It is interesting to discuss the influence from the sales learning curve. From Table 2, we find that $\rho_{i}^{t}, \rho_{i}^{I}, \Pi_{S}, \Pi_{R}, \Pi_{C}, t_{\max }, t_{\min }$ will increase and $T^{t}, T^{t}$ will decrease as the value of sales learning curve $\theta$ increases. The appropriate range $\left(t_{\min }, t_{\max }\right)$ will become winder upon increasing $\theta$.

Table 2. The influence of sales learning curve

\begin{tabular}{ccccccc}
\hline \multicolumn{7}{c}{ Decentralized decision model } \\
\hline$\theta_{i}$ & $\rho_{1}^{t}$ & $\rho_{2}^{t}$ & $T^{t}$ & $\Pi_{S}$ & $\Pi_{R}$ & $\Pi_{C}$ \\
\hline 0.01 & 1.242 & 1.190 & 0.1609 & 5709.61 & 6505.27 & 12214.9 \\
\hline 0.05 & 1.247 & 1.194 & 0.1607 & 5731.20 & 6519.03 & 12250.2 \\
\hline 0.09 & 1.252 & 1.198 & 0.1604 & 5753.08 & 6432.96 & 12286.1 \\
\hline \hline \multicolumn{7}{c}{ Centralized decision model } \\
\hline$\theta_{i}$ & $\rho_{1}^{I}$ & $\rho_{2}^{I}$ & $T^{I}$ & $\Pi_{C}$ & $t_{\max }$ & $t_{\min }$ \\
\hline 0.01 & 1.426 & 1.351 & 0.1708 & 12645.5 & 7.602 & 3.843 \\
\hline 0.05 & 1.435 & 1.358 & 0.1702 & 12689.4 & 7.709 & 3.896 \\
\hline 0.09 & 1.444 & 1.365 & 0.1697 & 12733.9 & 7.815 & 3.950 \\
\hline
\end{tabular}

\section{CONCLUSION}

This paper examines a multi-item distribution channel subject to supplier's credit period and retailer's promotional effort with sales learning curve. We determined the retailer's promotional effort for each item and the joint replenishment cycle in both decentralized and centralized decision models. We show that profits for both parties increase under centralized decision model when the credit period is kept within an appropriate range. The impacts of credit period and sales learning curve on the behavior of the channel have also been discussed. 


\section{REFERENCES}

1. H. Krishnan, R. K. Kapuscinski and D. A. Butz, Coordinating contracts for decentralized supply chain with retailer promotional effect, Management Science 50, 48-62, 2004.

2. J. Hahm and C. A. Yano, The economic lot and delivery scheduling problem: the common cycle case, IIE Transactions 27, 113-125, 1995.

3. M. Hammer, The superefficient company, Harvard Business Review 79, 82-91, 2001.

4. R. Rempala, Joint replenishment multiproduct inventory problem with continuous production and discrete demands, International Journal of Production Economics 81-82, 495-511, 2003.

5. T. H. Chen and J. M. Chen, Optimizing supply chain collaboration based on joint replenishment and channel coordination, Transportation Research Part E: Logistics and Transportation Review 41, 261-285, 2005.

6. S. K. Goyal, Economics order quantity under conditions of permissible delay in payments, Journal of the Operational Research Society 36(4), 335-338, 1985.

7. H. J. Chang, C. H. Hung and C. Y. Dye, An inventory model for deteriorating items with linear demand under condition of permissible delay in payments, Production planning and Control 12, 274-282, 2001.

8. J. T. Teng, On the economic order quantity under conditions of permissible delay in payments, Journal of the Operational Research Society 53, 915-918, 2002.

9. J. Kim, H. Hwang and S. Shinn, An optimal credit policy to increase supplier's profits with price-dependent demand functions, Production Planning \& Control 6(1): 45-50, 1995.

10. P. L. Abad and C. K. Jaggi, A joint approach for setting unit price and the length of the credit period for a seller when end demand is price sensitive, International Journal of Production Economics 83, 115-122, 2003.

11. G. J. Sheen and Y. C. Tsao, Channel coordination, trade credit and quantity discounts for freight cost, Transportation Research Part E: Logistics and Transportation Review 43(2), 112-128, 2007.

12. M. Leslie and C. A. Holloway, The sales learning curve, Harvard Business Review 84, 115-123, 2006.

13. W. L. Winston, Operations research applications and algorithms, CA: Thomson/ Brooks/Cole, Belmont, 2004. 